\title{
Trypanosoma culicavium sp. nov., an avian trypanosome transmitted by Culex mosquitoes
} Correspondence
Jan Votýpka
vapid@natur.cuni.cz

\author{
Jan Votýpka, ${ }^{1,2}$ Jana Szabová, ${ }^{1}$ Jana Rádrová, ${ }^{1}$ Lenka Zídková $^{1}$ \\ and Milena Svobodová ${ }^{1}$ \\ ${ }^{1}$ Department of Parasitology, Faculty of Science, Charles University in Prague, Vinicna 7, \\ Prague, CZ 128 44, Czech Republic \\ ${ }^{2}$ Biology Centre, Institute of Parasitology, Czech Academy of Sciences, Branisovska 31, \\ České Budějovice, CZ 370 05, Czech Republic
}

\begin{abstract}
A novel avian trypanosome, Trypanosoma culicavium sp. nov., isolated from Culex mosquitoes, is described on the basis of naturally and experimentally infected vectors and bird hosts, localization in the vector, morphological characters and molecular data. This study provides the first comprehensive description of a trypanosome species transmitted by mosquitoes, in which parasites form plugs and rosettes on the stomodeal valve. Trypanosomes occurred as long epimastigotes and short trypomastigotes in vectors and culture and as long trypomastigotes in birds. Transmission of parasites to bird hosts was achieved exclusively by ingestion of experimentally infected Culex mosquito females by canaries (Serinus canaria), but not by Japanese quails (Coturnix japonica), nor by the bite of infected vectors, nor by ingestion of parasites from laboratory cultures. Transmission experiments and the identity of isolates from collared flycatchers (Ficedula albicollis) and Culex mosquitoes suggests that the natural hosts of T. culicavium are insectivorous songbirds (Passeriformes). Phylogenetic analyses of small-subunit rRNA and glycosomal glyceraldehyde-3-phosphate dehydrogenase gene sequences demonstrated that $T$. culicavium sp. nov. is more related to Trypanosoma corvi than to other avian trypanosomes (e.g. Trypanosoma avium and Trypanosoma bennetti).
\end{abstract}

\section{INTRODUCTION}

Mosquitoes are among the most important vectors of human and animal pathogens (viruses, bacteria, protozoa and filariae) worldwide. However, relative to other biting flies, mosquitoes have been poorly studied as vectors of trypanosomes (genus Trypanosoma; family Trypanosomatidae; order Kinetoplastea), widespread blood parasites of vertebrates. Although several well-known trypanosome species are causative agents of important diseases (e.g. Chagas disease, sleeping sickness and nagana), the majority of extant trypanosome species remains overlooked. Avian trypanosomes, for instance, are a much less well-known group of trypanosomes, even though almost 100 trypanosome species

\footnotetext{
Abbreviations: gGAPDH, glycosomal glyceraldehyde-3-phosphate dehydrogenase; p.i., post-infection; SSU, small subunit.

The GenBank/EMBL/DDBJ accession numbers for the SSU rRNA gene sequences reported in this paper are H0107966-HO107970, H0909083 and H0909084 for Trypanosoma culicavium sp. nov. isolates PAS109, CUL28, CUL31, PAS99, CUL6, CUL24 and CUL30, respectively. Those for the gGAPDH gene sequences reported in this paper are H0.906657-H0.906661 for T. culicavium sp. nov. isolates CUL1, PAS99 and PAS109, T. corvi OA6 and T. avium ANI14A, respectively.

A supplementary table is available with the online version of this paper.
}

have been described to date (Baker, 1976; Bennett et al., 1982; Podlipaev, 1990; Sehgal et al., 2001). The biology of avian trypanosomes (vectors, life cycles, host and vector specificity) is poorly known or totally unknown, despite the fact that some species could be pathogenic to their host (Molyneux et al., 1983).

Experimental transmission of parasites belonging to the Trypanosoma avium clade from insect vectors to bird hosts has been achieved using the blackflies Eusimulium latipes (Votýpka \& Svobodová, 2004) and Simulium rugglesi (Desser et al., 1975), whereas the hippoboscid fly Ornithomyia avicularia was shown to transmit parasites of the Trypanosoma corvi clade (Baker, 1956; Mungomba et al., 1989). Vectors of avian trypanosomes from the Trypanosoma bennetti clade are still unknown. Although mosquitoes have been considered several times as vectors of avian trypanosomes (for the first time in 1904 by Schaudinn, who observed division of epimastigotes in the midgut and hindgut of Culex pipiens females blood-fed on an infected owl), and several transmission experiments involving mosquitoes and birds have been performed (David \& Nair, 1955; Bennett, 1961; Chatterjee, 1977), the role of mosquitoes in the transmission of avian trypanosomes in nature has not yet been demonstrated unambiguously. 
In dixenous trypanosomatids, parasite transmission is accomplished either by the bite of blood-sucking vectors, in which parasites develop in the fore part of the alimentary tract (e.g. leishmanias in phlebotomine sandflies) or in salivary glands (e.g. Trypanosoma brucei in tsetse flies), or by the contamination of host skin and/or conjunctiva by vector faeces during and after blood sucking of vectors, where parasites develop in the hind part of the vector's digestive tract (e.g. Trypanosoma cruzi in kissing bugs). Much less is known about the transmission of avian trypanosomes, some of which accomplish transmission by ingestion of infected vectors or the contamination of abraded skin or host conjunctiva by parasites present in vector faeces (the hippoboscid fly Ornithomyia avicularia and blackfly Eusimulium securiforme) (Baker, 1956; Mungomba et al., 1989; Desser et al., 1975; Votýpka \& Svobodová, 2004).

Since morphological identification of trypanosomes is impossible (Desquesnes \& Dávila, 2002), molecular techniques have been used to identify trypanosomes from a number of animals. According to recent molecular phylogenetic studies (Votýpka et al., 2002, 2004; Hamilton et al., 2007), bird trypanosomes form three distinct clades named after the principal species: T. avium, T. corvi and T. bennetti. Several recent studies have presented data on the occurrence of avian trypanosomes in blackflies, hippoboscid flies and mosquitoes (Votýpka et al., 2002, 2004; Votýpka \& Svobodová, 2004; Van Dyken et al., 2006). Species identification of avian trypanosomes and identification of their host range require matching the trypanosomes found in vectors with those in vertebrate hosts. In our previous study (Votýpka et al., 2002), we compared small-subunit (SSU) rRNA gene sequences to confirm ornithophilic simuliids as probable vectors of bird trypanosomes, which was later confirmed experimentally (Votýpka \& Svobodová, 2004). Moreover, one mosquito isolate marked as CUL1 belonged to the genus Trypanosoma, but was not similar to T. avium sensu stricto isolated from blackflies (Votýpka et al., 2002). In this study, we formally describe a novel trypanosome species, Trypanosoma culicavium sp. nov., compare it with other avian and mosquito trypanosomes and elucidate its mode of transmission from the insect vector to the bird host.

\section{METHODS}

Detection of parasites from mosquitoes and birds. Mosquitoes were collected in the framework of two independent studies. In the first study, in 1998-2002, dissection to determine the presence of trypanosomes and subsequent cultivation was carried out on mosquitoes collected at several localities in the Czech Republic (Prague, southern Moravia and southern Bohemia). In the second study, in 2005-2007, using mosquitoes collected in southern Bohemia and southern Moravia, trypanosomes in mosquitoes were detected by PCR only.

Bird blood samples were taken from adult collared flycatcher (Ficedula albicollis) females trapped in nest-boxes during the breeding season in the Milovický forest, southern Moravia. Methods of mosquito collection and dissection, bird blood sampling and parasite isolation used in the present study have been described elsewhere (Votýpka et al., 2002, 2008; Votýpka \& Svobodová, 2004).
RAPD and phylogenetic analyses. Total DNA samples were extracted from cultured trypanosome lines using a DNA tissue isolation kit (Roche) according to the manufacturer's instructions. DNA samples of mosquito isolates were evaluated by RAPD analysis as described by Svobodová et al. (2007). SSU rRNA gene loci of selected isolates were sequenced by using universal eukaryotic primers Medlin A (5'-CTGGTTGATCCTGCCAG-3') and Medlin B (5' -TGATCCTTCTGCAGGTTCACCTAC-3'). Glycosomal glyceraldehyde-3phosphate dehydrogenase ( $\mathrm{gGAPDH})$ genes from the isolates were amplified using primers G3 (5'-TTYGCCGYATYGGYCGCATGG-3') and G4a (5'-GTTYTGCAGSGTCGCCTTGG-3') as described by Hamilton et al. (2004). The PCR products of both genes were purified and sequenced directly. A dataset containing 43 gGAPDH sequences of trypanosomatids was created, including sequences of T. culicavium.

Phylogenetic analyses of the two analysed genes (including isolate CUL1, of which the SSU rRNA gene sequence was published previously under GenBank accession no. AF416561) were performed as described by Zídková et al. (2010); phylogenetic trees were reconstructed using maximum-parsimony (PAUP* 4.0b10), maximum-likelihood (PhyML win32) and Bayesian (MrBayes 3.1.1) methods. Sequences used for phylogenetic analyses are detailed in Supplementary Table S1, available in IJSEM Online.

PCR screening for trypanosomes in mosquitoes. For PCR screening of trypanosomes, calculation of prevalence and phylogenetic analyses, we used protocols described by Van Dyken et al. (2006) and Votýpka et al. (2008). Mosquitoes were sorted into species and unfed females were frozen at $-70{ }^{\circ} \mathrm{C}$ in pools of $10-50$ for later processing. Pooled females were homogenized in RNAlater RNA stabilization reagent (Qiagen) and total DNA was extracted as described above.

Pooled female mosquitoes were tested for the presence of trypanosomes using specific trypanosome PCR primers to amplify $18 \mathrm{~S}$ rRNA gene. Primers used for amplification were R-221 (5'-GGTTCCTTTCCTGATTTACG-3') and Medlin B (5'-TGATCCTTCTGCAGGTTCACCTAC- $\left.3^{\prime}\right)$. As a negative control, DNA from a laboratory colony of Culex quinquefasciatus was used. Amplicons of 1300$1400 \mathrm{bp}$ were gel-isolated and directly sequenced. Homologous $18 \mathrm{~S}$ rRNA gene sequences originated from our previous studies (Votýpka et al., 2002, 2004) or were downloaded from the NCBI database. Newly obtained sequence data were aligned and compared using CLUSTAL_X version 1.81 to find the most closely related trypanosome $18 \mathrm{~S}$ rRNA gene sequences.

Experimental infection of mosquitoes and transmission to birds. All animal experiments were performed according to Czech law and Guidelines on Animal Experimentation. Trypanosome isolate CUL1 from mosquito was used for infectious experiments with mosquitoes as well as with birds; bird isolate PAS99 was used only for infection of mosquitoes. In several independent experiments, 10- to 14day-old adult female mosquitoes of the laboratory reared colony of $C$. quinquefasciatus (a member of the Culex pipiens complex) were infected by feeding through a chick skin membrane on heat-inactivated rabbit blood containing a 7 - to 14 -day-old culture of $10^{6}-10^{7}$ CUL1 parasites $\mathrm{ml}^{-1}$. In a single experiment, the same procedure was followed with $C$. quinquefasciatus and isolate PAS99 and with Aedes aegypti and isolate CUL1 ( $10^{7}$ parasites $\mathrm{ml}^{-1}$ in both experiments). Fed females were separated 1 day after blood feeding. Mosquitoes were maintained in cages at $18-20{ }^{\circ} \mathrm{C}, 80 \%$ relative humidity and supplemented with $50 \%$ sucrose solution. Mosquito females were dissected 12-28 days postinfection (p.i.) and examined under a light microscope for determination of the location and density of flagellates in the gut.

Three different methods were used for experimental infection of laboratory reared canaries (Serinus canaria, Passeriformes) and Japanese quails (Coturnix japonica, Galliformes): (i) by bite of experimentally infected mosquitoes, (ii) by ingestion of experimentally 
infected mosquitoes and (iii) by ingestion of trypanosome culture forms (see Table 1). All experimental birds were negative for trypanosomes before experimental infections.

In method (i), mosquito females, 10-20 days after the first infectious feeding, were allowed to feed again on nine canaries and five Japanese quails. Immediately after this second feeding, fed females were separated and dissected and their guts and stomodeal valves were checked microscopically. In method (ii), guts from infected mosquitoes, 2-3 weeks after infection, were homogenized in saline and were applied perorally to canaries and Japanese quails. In method (iii), canaries and Japanese quails were inoculated perorally with $10^{7}$ trypanosomes from a 7-day-old culture.

The presence of trypanosomes in experimental birds was checked at 15, 40, 60 and 240 days p.i. by in vitro cultivation of blood on SNB-9 blood agar and by xenodiagnosis. For Japanese quails, the experiments were terminated at 60 days p.i., and their femur bone marrow was used for cultivation on SNB-9 blood agar. For xenodiagnosis and a transmission cycle investigation, naive mosquito females of C. quinquefasciatus were allowed to suck blood on experimentally infected canaries and Japanese quails 3 weeks after experimental infection. Fed females were separated 1 day after blood feeding and were dissected and microscopically inspected 9 days after blood-feeding.

Light and electron microscopy. Light-microscope preparations were made from cultured cells (a 5- to 7-day-old culture in exponential phase) of isolates CUL1, CUL6, CUL30, PAS99 and PAS109 (T. culicavium) and ITMAP and OA6 (T. corvi), on cells (CUL1) extracted from experimentally infected mosquitoes during their dissection (10 days p.i., when a majority of parasites are attached to the stomodeal valve) and from blood films prepared from birds experimentally infected by the CUL1 strain (30 days p.i.; only two trypanosomes were seen). For parasite measurements, the QuickPHOTO MICRO software (Olympus) and Grmeasure software, CCD camera DP70 and Olympus BX51 microscope were used. Morphometric data of cell length were obtained for 25 cells of each morphotype: cultured cells of all studied isolates and trypanosomes from experimentally infected mosquitoes in the case of CUL1. Measurements (in $\mu \mathrm{m}$ ) included cell length and (for CUL1) cell width at the widest point and length of the free flagellum. Different morphological types from culture and experimentally infected mosquitoes were compared using ANOVA post-hoc comparisons (Statistica software). Electron-microscope preparations from 5- to 7-day-old cultures and measurement of the kinetoplast thickness of the mosquito (CUL6) and bird (PAS99) isolates were made as described by Votýpka et al. (2002).

\section{RESULTS AND DISCUSSION}

\section{Origin and prevalence of Trypanosoma culicavium sp. nov. in mosquitoes}

Trypanosoma culicavium (isolate ICUL/CZ/1998/CUL1) was isolated from the stomodeal valve of a Culex pipiens
(Culicidae, Diptera) mosquito female captured in southern Moravia (Milovický forest: $48^{\circ} 50^{\prime} \mathrm{N} 16^{\circ} 42^{\prime} \mathrm{E}, 290 \mathrm{~m}$ above sea level) in 1998. Altogether, 1032 specimens of mosquito females (899 of C. pipiens and 133 of Culex modestus) captured in southern Moravia, southern Bohemia and the vicinity of Prague city were dissected, and 31 kinetoplastid strains were established. Of those, 23 strains were $T$. culicavium according to RAPD analysis (data not shown). Twenty-two isolates originated from $C$. pipiens (prevalence $2.4 \%$ ) and one (CUL30) from C. modestus (prevalence $0.7 \%) ; 14$ isolates originated from Prague, five from southern Moravia and four from southern Bohemia. In the next step, six of these isolates [one Prague isolate (CUL24), two Moravian isolates (CUL1 and CUL6) and three southern Bohemia isolates (CUL28, CUL30 and CUL31)] were sequenced and shown to be identical to $T$. culicavium by sequence analyses of the SSU rRNA gene (see Fig. 4b; Table 2). Avian trypanosomes are transmitted by diverse vectors; however, the mere presence of trypanosomes in a blood-sucking invertebrate does not necessarily incriminate it as a vector. Only if parasites survive defecation of the rest of a blood-meal and successfully colonize a digestive tract (or salivary glands) can we speculate about transmission to vertebrate hosts. In our case, mature infections were localized to the stomodeal valve, and all naturally infected mosquito females had no blood in their intestine.

In conjunction with trypanosome PCR screening among mosquitoes captured around fishponds in southern Moravia and Bohemia, 129 pools representing 2490 individuals of Culex (Culex) pipiens, 3034 of Culex (Barraudius) modestus and 41 of Culex (Culex) torrentium were tested. Altogether, 11 pools were positive for trypanosome DNAs and, of those, only seven $(5.5 \%$ of the total) were $T$. culicavium by sequencing of part of the SSU rRNA gene. It can be supposed that only a single specimen within each positive pool was infected. The prevalence of tested mosquitoes was $0.3 \%$ for C. pipiens and $0.05 \%$ for $C$. modestus. All specimens of $C$. torrentium were negative. Since the PCR-tested mosquitoes had no blood in their guts, we suppose that mosquito females had not been simply carrying replicating trypanosomes in an undigested blood-meal.

The presence of parasites found by dissection and by PCR demonstrates that T. culicavium occurs in Culex mosquitoes (C. pipiens and C. modestus) at various localities (forest areas and wetlands) in Bohemia and Moravia. Our data, based on

Table 1. Set-up of experimental transmission of trypanosomes to bird hosts

\begin{tabular}{|lccc|}
\hline Route of infection & Infection dose & Canaries $^{*}$ & Quails $^{*}$ \\
\hline (i) By bite & $2-14$ infectious Culex & $9 / 0$ & $5 / 0$ \\
(ii) Perorally & $7-15$ infectious Culex & $8 / 3$ & $4 / 0$ \\
(iii) Perorally & $10^{7}$ culture forms & $4 / 0$ & $3 / 0$ \\
\hline
\end{tabular}

${ }^{*}$ Numbers of experimental/positive birds. 
Table 2. Isolates of $T$. culicavium originating from Culex mosquitoes and collared flycatchers

\begin{tabular}{|llllll|}
\hline Isolate & Host species & \multicolumn{1}{c|}{ Locality } & Year & \multicolumn{2}{c|}{ GenBank accession no. } \\
\cline { 5 - 6 } & & & & SSU rRNA gene & gGAPDH \\
\hline CUL1 & Culex pipiens & Southern Moravia & 1998 & AF416561 & HQ906657 \\
CUL6 & Culex pipiens & Southern Moravia & 2000 & HQ107970 & ND \\
CUL24 & Culex pipiens & Prague & 2001 & HQ909083 & ND \\
CUL28 & Culex pipiens & Southern Bohemia & 2002 & HQ107967 & ND \\
CUL30 & Culex modestus & Southern Bohemia & 2002 & HQ909084 & ND \\
CUL31 & Culex pipiens & Southern Bohemia & 2006 & HQ107968 & HQ906658 \\
PAS99 & Ficedula albicollis & Southern Moravia & 2006 & HQ107969 & HQ906659 \\
PAS109 & Ficedula albicollis & Southern Moravia & 2007 & HQ107966 & \\
\hline
\end{tabular}

ND, Not determined.

naturally infected mosquitoes, indicate that $T$. culicavium is able to infect at least two different subgenera of the genus Culex. The three- to sixfold lower prevalence of $T$. culicavium in C. (Barraudius) modestus could indicate that mosquitoes from the subgenus Culex (e.g. C. pipiens) are more susceptible hosts for T. culicavium than mosquitoes of other subgenera, or that these species feed on different hosts (J. Votýpka, unpublished results).

Molecular detection of avian trypanosomes (belonging to the T. avium and T. corvi groups) in wild-caught vectors was reported by Van Dyken et al. (2006), who found three $(0.2 \%)$ positive Culex pipiens and Culex (Culex) tarsalis mosquitoes.

\section{Experimental infections and transmissions}

For several infection experiments with isolate CUL1, more than 1000 mosquito females from a laboratory reared colony of C. quinquefasciatus were used. The infection rate after blood feeding through a chicken-skin membrane was $60-85 \%$. Trypanosomes were localized mainly on the stomodeal valve, and the majority of positive mosquito females were heavily infected (i.e. more than 1000 parasites per gut). Thus, C. quinquefasciatus was shown to be a good experimental host of T. culicavium. To prove transmissibility of avian isolates of T. culicavium, 70 females of $C$. quinquefasciatus were fed with bird isolate PAS99. After 14-28 days, 26 mosquitoes (37\%) were found to be infected with parasites attached to the stomodeal valve.

To assess the vector specificity of T. culicavium, 92 females of A. aegypti were fed with CUL1 isolate. Only two specimens $(2 \%)$ were found to be positive with parasites attached to the stomodeal valve, one of them being heavily infected. Control C. quinquefasciatus females fed on the same inoculum of trypanosomes were infected in $66 \%$ of cases. This demonstrates that genera other than Culex (the natural hosts) are able to become infected under experimental conditions, albeit with a very low infection rate. Information about vector specificity of avian trypanosomes is very limited and inconsistent (see David \& Nair, 1955;
Baker, 1956; Bennett, 1961, 1970; Chatterjee, 1977; Miltgen \& Landau, 1982; Van Dyken et al., 2006; Reeves et al., 2007). We suppose that T. culicavium is not identical to a trypanosome from Aedes mosquitoes described by David \& Nair (1955), which occurred in the hindgut. The ability of $T$. culicavium to develop in the intestine of Culex mosquitoes from two subgenera (Culex and Barraudius) indicates a host range that extends over different mosquito subgenera, but probably not genera.

The development of avian trypanosomes has been demonstrated in several dipteran species. In blackflies, epimastigotes replicate during blood-meal digestion in the midgut and, after peritrophic membrane rupture, the epimastigotes migrate to the hindgut and rectum, where they attach to a chitinous intima via hemidesmosomes (Bennett, 1970; Desser, 1977; Dirie et al., 1990; Votýpka \& Svobodová, 2004). The transmission of parasites to a new host can occur via vector ingestion or defecation, and possibly via urination (diuresis) (Dirie et al., 1990; Votýpka \& Svobodová, 2004). Transmission by vector ingestion was also demonstrated for hippoboscid flies by Baker (1956) and hemidesmosome-like adhesion for trypanosomes in the posterior part of the alimentary tract of Ornithomyia avicularia was reported by Mungomba et al. (1989).

T. culicavium (isolate CUL1) was shown to occur first in the mosquito midgut; later, the parasite moves to the anterior part of the gut, attaches to the stomodeal valve (Fig. 1) and degrades it in a way similar to leishmania parasites in sandfly vectors (Volf et al., 2004). It has been speculated that the mode of transmission to birds is probably regurgitative, the ingested blood and trypanosomes being regurgitated, as a result of the less functional proventriculus, back into the bite wound during feeding (Volf et al., 2004).

Our experiments, focused on the transmission mode of trypanosomes from insect vectors to bird hosts, resulted in successful transmission only in the case of experimentally infected Culex mosquitoes ingested by canaries; all birds inoculated with trypanosome stages from culture or bitten by infected mosquitoes were negative (Table 1). 

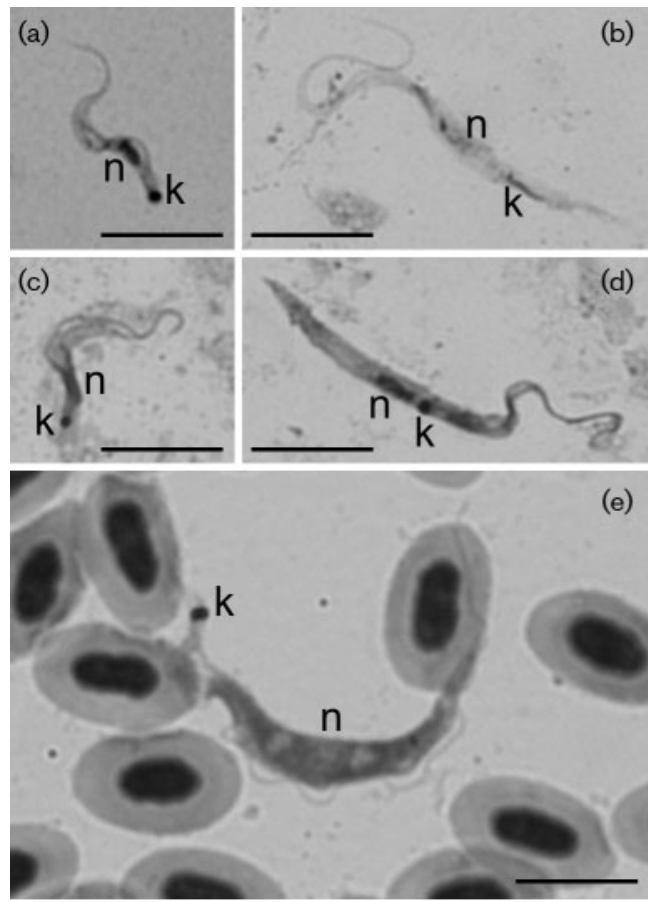

(b)

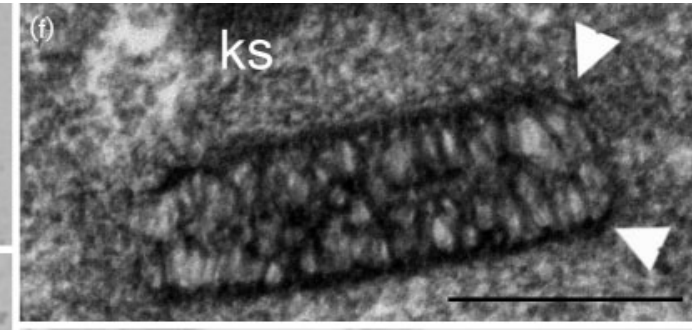

(g)
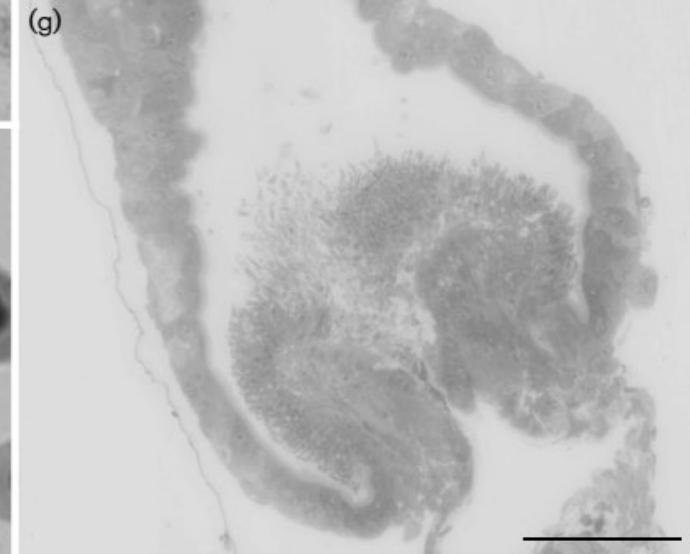

Fig. 1. (a-e) Micrographs of Giemsa-stained Trypanosoma culicavium showing short trypomastigote (a, c) and long epimastigote $(b, d)$ forms from the stomodeal valve of experimentally infected Culex mosquito females 10 days after infection $(a, b)$ and from a 7-day-old culture (c, d) and long trypomastigote from the blood of an experimentally infected canary at 30 days p.i. (e). (f) Transmission electron micrograph of kinetoplast with the characteristic low-pitched and elongated shape. k, Kinetoplast; ks, kinetosome of the flagellum; n, nucleus. Arrowheads indicate the thickness of the kinetoplast. (g) Semi-thin section of the stomodeal valve with attached parasites in experimentally infected Culex mosquito females, 10 days p.i. Bars, $10 \mu \mathrm{m}(\mathrm{a}-\mathrm{e}), 0.5 \mu \mathrm{m}(\mathrm{f})$ and $50 \mu \mathrm{m}(\mathrm{g})$.

Trypanosomes were found by xenodiagnosis in the blood of three of eight canaries that were infected perorally with gut forms of parasites from experimentally infected mosquito females. In these three positive canaries, the infection rates of the xenodiagnosis were $82 \%$ ( 23 of 28 specimens; 45 days p.i.), $10 \%$ ( 1 of $10 ; 17$ days p.i.) and $8 \%$ ( 1 of $12 ; 22$ days p.i.). The mosquito females were dissected after defecation to prove that the infection persisted after complete digestion of the blood-meal, and parasites were found in their stomodeal valves in all cases. The presence of trypanosomes was confirmed by in vitro cultivations only in one xenodiagnosis-positive canary (with the highest mosquito infection rate, of $82 \%$ ), and parasites were detected from day 15 to day 40 p.i. Later cultivations were negative, probably because of the disappearance of trypanosomes from peripheral blood.

The changes of the mosquito stomodeal valve resemble those seen in sandflies, where they are suggested to facilitate transmission when biting (Volf et al., 2004). Therefore, we originally supposed that $T$. culicavium was transmitted by mosquito bite. In order to find out which morphological forms of T. culicavium are regurgitated, infected mosquitoes were force-fed using a Hertig apparatus (Hertig \& McConnell,
1963); however, no trypanosomes were regurgitated. Moreover, after feeding infected mosquitoes on a chick-skin membrane, no trypanosomes were found in the blood used for feeding. Other indirect evidence came from our field studies. Buzzards were readily attacked by Culex mosquitoes and, although the prevalence of trypanosomes in mosquitoes was as high as $10 \%$, T. culicavium was never isolated from the blood of raptors (J. Votýpka and others, unpublished results; Černý et al., 2011). These results, together with transmission experiments to canaries as well as the isolation of T. culicavium exclusively from mosquito-eating passerines, suggests that inoculation by bite does not play a significant role in natural transmission and that $T$. culicavium is indeed transmitted in the first instance by ingestion of infected mosquitoes.

Our experiments proved unambiguously that $T$. culicavium (CUL1 isolate) is transmissible to Culex mosquitoes by feeding on an infected bird and demonstrated the successful completion of the T. culicavium life cycle. In addition, similar infection rates of Culex mosquitoes infected by either artificial feeding through a chicken skin membrane (70$85 \%$ ) or by naturally feeding on an infected bird host (up to $82 \%$ ) demonstrate that the prevalence in experimentally infected mosquitoes mimics natural infection. 


\section{Isolation of T. culicavium from wild bird hosts}

Our results based on experimental infections and transmission mode suggested the role of insectivorous songbirds in the life cycle of $T$. culicavium. Collared flycatchers (Ficedula albicollis) are reported to feed on mosquitoes (Hudec \& Št'astný, 2005), and 21 of 56 sampled birds were positive for Trypanosoma sp. Twelve isolates were established; of these, two isolates (PAS99 and PAS109) were identical to T. culicavium (isolate CUL1) as shown by sequencing of the SSU rRNA gene and gGAPDH (see Fig. 4; Table 2).

The transmission of $T$. culicavium under laboratory conditions occurs by ingestion and this, together with the fact that flycatcher isolates (PAS99 and PAS109) are identical to CUL1 by sequencing, suggests that the natural hosts of T. culicavium are insectivorous songbirds (Passeriformes).

\section{Light microscopy and transmission electron microscopy}

Two morphologically different types of trypanosome (isolate CUL1) were present on slides obtained from mosquito stomodeal valves as well as on slides from in vitro cell cultures (isolates CUL1, CUL6, CUL30, PAS99 and PAS109). Trypomastigotes were the shorter observed morphotypes, whereas the longer morphotypes were epimastigotes (Fig. 1). For isolate CUL1, body lengths of short $(12.0 \pm 0.6 \mu \mathrm{m}$, $n=25)$ as well as long $(24.3 \pm 2.6 \mu \mathrm{m}, n=25)$ insect forms were significantly shorter $\left(\right.$ ANOVA; $F_{(1,50)}=29.81 ; P<0.001$ and $\left.\mathrm{F}_{(1,50)}=36.66 ; \quad P<0.001\right)$ than culture forms of trypomastigotes $(13.5 \pm 0.7 \mu \mathrm{m}, n=25)$ and epimastigotes $(30.8 \pm 3.7 \mu \mathrm{m}, n=25)$, respectively (Fig. 2). Significant differences were not found among lengths of cultured cells

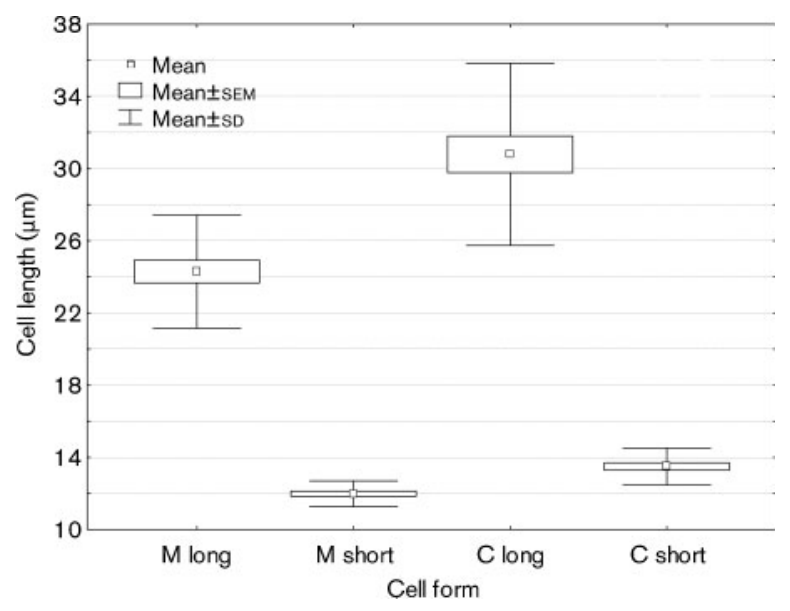

Fig. 2. Comparison of cell lengths of long and short forms of $T$. culicavium isolate CUL1 originating from experimentally infected C. quinquefasciatus mosquito females (M) and from culture (C). Twenty-five cells of each type were measured. of five isolates of T. culicavium (CUL1, CUL6, CUL30, PAS99 and PAS109), nor for the short trypomastigote form (ANOVA; $\left.\mathrm{F}_{(4,125)}=0.19 ; P=0.94\right)$ nor for the long epimastigote form (ANOVA; $\mathrm{F}_{(4,125)}=1.09 ; P=0.36$ ).

Baker (1966) observed similar morphotypes ('big epimastigotes and small trypomastigotes') in a culture of trypanosomes obtained from a rook. In contrast, we observed only one morphotype (epimastigotes) in a culture of T. corvi (isolate OA6 and LSHTM/ITMAP 180795), and the cell length was significantly different $\left(\right.$ ANOVA; $F_{(2,300)}=450.72$; $P<0,001$ ) from that of $T$. culicavium (shorter than epimastigotes and longer than trypomastigotes; Fig. 3). It is possible that Baker worked initially with T. culicavium (which exhibited two morphotypes); nevertheless, the strain originated from a rook and deposited as LSHTM belongs to T. corvi, with one morphotype only.

Only two trypomastigotes were found on a Giemsa-stained thin blood smear of experimentally infected canary (Fig. 1). The utility of delineating trypanosome species based on morphometrics of blood-stream forms is, however, questionable, as pleomorphism of numerous trypanosomes has been documented (Baker, 1976).

Culture forms of $T$. culicavium were also examined by transmission electron microscopy (Fig. 1). We have shown previously (Lukeš \& Votýpka, 2000) that the kinetoplast thickness and the minicircle size vary significantly among avian trypanosomes (e.g. T. corvi, $0.37 \mu \mathrm{m} ; \mathrm{T}$. avium, $0.69-0.85 \mu \mathrm{m})$. The kinetoplast thickness of T. culicavium mosquito isolate CUL1 $[0.310 \pm 0.031 \mu \mathrm{m} ; n=35$; see our previous study (Votýpka et al., 2002)] was similar to that of newly measured bird (PAS99) and mosquito (CUL6) isolates [respectively $0.331 \pm 0.045 \mu \mathrm{m}(n=38)$ and $0.299 \pm$ $0.023 \mu \mathrm{m}(n=40)]$, confirming the identity of the isolates.

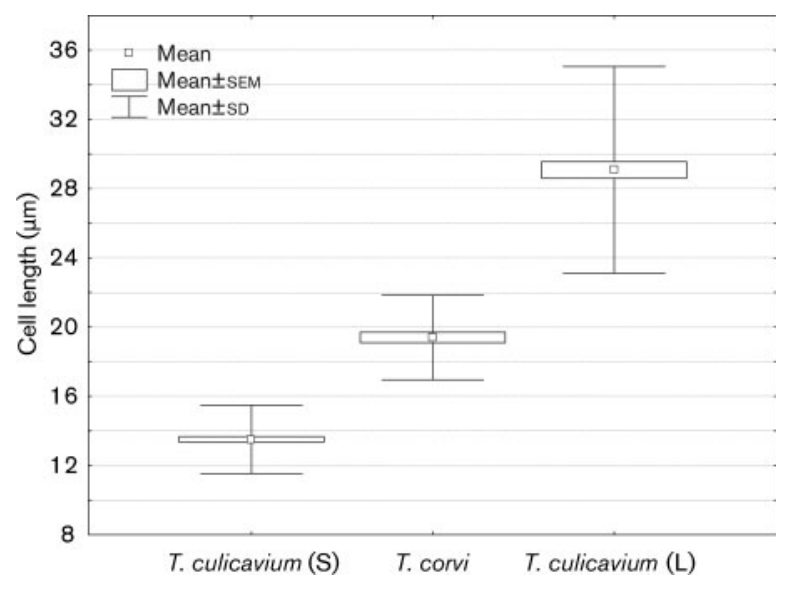

Fig. 3. Comparison of cell lengths of long (L) and short (S) forms of $T$. culicavium isolates CUL1, CUL6, PAS99 and PAS109 and T. corvi isolates OA6 and LSHTM. Twenty-five cells of each type and isolate were measured. 


\section{Phylogenetic position and comparison with other described species}

As shown previously (Votýpka et al., 2002, 2004), SSU rRNA gene nucleotide sequence analysis indicated that the newly described species of the genus Trypanosoma (represented by isolate CUL1) clusters with trypanosomes isolated from birds [isolate LSHTM/ITMAP 180795 from rook (Corvus frugilegus) caught in the UK] and from a hippoboscid fly [isolate OA6 (Ornithomyia avicularia) captured in the Czech Republic], which together form a highly supported T. corvi clade. A new phylogenetic tree (Fig. 4) was created on the basis of gGAPDH and SSU rRNA gene sequences, containing all relevant GenBank sequences and sequences of our isolates of T. culicavium obtained from mosquitoes (Culex pipiens; CUL1 and CUL6 from southern Moravia, CUL28, CUL30 and CUL31 from southern Bohemia and CUL24 from Prague) and two isolates from collared flycatchers (Ficedula albicollis; PAS109 and PAS99) captured in southern Moravia. Both groups of isolates, from mosquitoes as well as from birds, formed a highly supported and separate clade together with reference isolate CUL1, closely related to T. corvi. The relationship between T. culicavium and the $T$. corvi clade was shown unambiguously by a phylogenetic tree based on the SSU rRNA gene sequence (Fig. 4b).

So far, only two species of trypanosomes have been described to occur in mosquitoes. The first is Trypanosoma rotatorium (Mayer, 1843), which parasitizes several frog species and is transmitted by Aedes or Culex mosquitoes. This species is well known and, together with other trypanosomes isolated from amphibians and fish, belongs to a well-supported aquatic clade (Votýpka et al., 2002, 2004; Hamilton et al., 2007). The second species is the avian trypanosome Trypanosoma noctuae (Schaudinn, 1904) described from the little owl (Athene noctua) in Europe. C. pipiens was mentioned in the original description as a vector. Notably, there are several uncertainties in the original description, and Schaudinn (1904) erroneously supposed that the parasite has a sexual phase in the mosquito ovary, assuming that the parasite is congenitally transmitted among mosquitoes. To exclude any possibility of transovarial or transstadial transmission among mosquitoes (without a vertebrate host), several additional laboratory experiments were carried out. Of 197 dissected adult C. quinquefasciatus mosquitoes (166 females and 31 males) hatched from eggs oviposited by 12 infected females and reared in water containing dead infected females, we did not record any positive cases.

According to Schaudinn's account, he was dealing with two separate parasites of the little owl, T. noctuae and Trypanosoma ziemanni (later synonymized with Leucocytozoon danilewskyi). The genus Trypanomorpha (as well as family Trypanomorphidae) was established by Woodcock (1906) and the only known species so far is the type species, Trypanomorpha noctuae; however, the family and genus are not generally accepted nowadays. It is impossible to compare our results with the records of Schaudinn (1904), due to the absence of any relevant description. It has been suggested that the parasite described by Schaudinn from mosquitoes represented monoxenous trypanosomatids of the genus Crithidia (Novy et al. 1907), and that the parasites isolated by Novy et al. (1907) from Culex mosquitoes and originally described as 'Trypanosoma (Herpetomonas) culicis, n. sp'. represented monoxenous trypanosomatids of the genus Blastocrithidia (Wallace \& Johnson, 1961).

We therefore describe here a new species of the genus Trypanosoma. Trypanosoma culicavium sp. nov. is the first named avian trypanosome species to be described from mosquitoes, and the species characters are based on a combination of vector specificity, morphology and, particularly, molecular phylogeny. We suppose that the avian trypanosomes $T$. corvi (isolated from rooks and strictly ornithophilic hippoboscid flies) and T. culicavium (from flycatchers and mosquitoes) are different but closely related species. Both the transmission of T. culicavium to canaries by ingestion of infected Culex mosquitoes and the similarity of trypanosomes obtained from blood of collared flycatchers (F. albicollis) to isolates from Culex mosquitoes suggest insectivorous songbirds as the natural vertebrate hosts of T. culicavium.

\section{Taxonomic summary (based on isolate CUL1)}

Diagnosis. Two different morphotypes, short trypomastigotes and long epimastigotes, were observed in the mosquito host as well as in cell culture. Insect forms of experimentally infected Culex mosquitoes were $12.0 \pm 0.6$ $\mu \mathrm{m} \quad(n=25)$ and $24.3 \pm 2.6 \mu \mathrm{m} \quad(n=25)$ long and $1.2 \pm$ $0.2 \mu \mathrm{m}(n=25)$ and $1.7 \pm 0.3 \mu \mathrm{m}(n=25)$ wide with flagella that were $5.6 \pm 0.6 \mu \mathrm{m}(n=25)$ and $9.3 \pm 1.1 \mu \mathrm{m}(n=25)$ long, respectively. The nucleus was situated in the middle of the cell with the kinetoplast next to it. The thickness of the kinetoplast was $0.310 \pm 0.031 \mu \mathrm{m}(n=35)$.

Trypomastigotes in the blood of experimentally infected canaries were narrow, slightly curved, with a tapering and pointed posterior end. A narrow undulating membrane was located on the external side of the curvature. The length of the body was $40.7-40.9 \mu \mathrm{m}$, the width was $3.7-$ $4.1 \mu \mathrm{m}$ and a free, relatively short flagellum varied in length from 6.8 to $8.0 \mu \mathrm{m}$.

Type host (vector). Female of mosquito Culex pipiens (Linnaeus, 1758).

Additional host (vector). Female of mosquito Culex modestus (Ficalbi, 1890).

Experimental invertebrate host (vector). Female of mosquito Culex quinquefasciatus (Say, 1823).

Localization in type host (vector). Stomodeal valve (mature infection). 
(a)

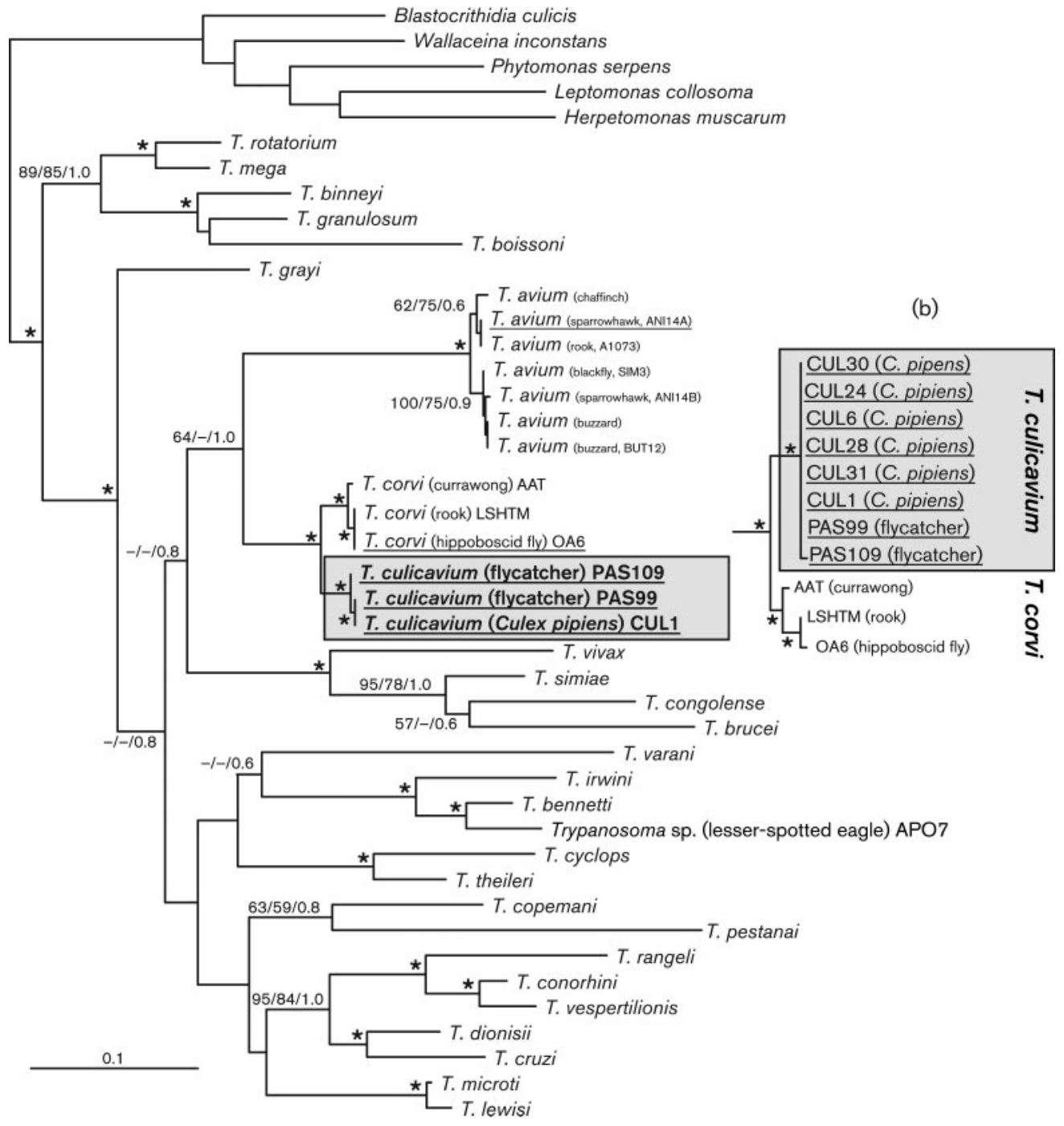

Fig. 4. (a) Maximum-likelihood phylogenetic tree inferred from partial gGAPDH gene sequences including 43 taxa (GenBank accession numbers are indicated). The figure shows topologies obtained by maximum-likelihood using the GTR model for nucleotide substitutions with $\Gamma$ distribution in $8+1$ categories as implemented in PhyML; all parameters were estimated from the dataset. Bootstrap values from maximum-likelihood (1000 replicates) and maximum-parsimony (1000 replicates) and Bayesian support values (posterior probabilities; $5 \times 10^{6}$ generations) are shown at nodes (maximum-likelihood $/ \mathrm{maximum}$ parsimony/Bayesian); dashes (-) indicate bootstrap values below $50 \%$ or posterior probability below 0.5 or nodes with different topology. Asterisks $\left(^{*}\right)$ at nodes denote Bayesian posterior probabilities and bootstrap percentages of $95 \%$ or higher. Bar, 0.1 substitutions per site. (b) Part of the maximum-likelihood phylogenetic tree inferred from SSU rRNA gene sequences, including 55 taxa (including CUL1). New SSU rRNA gene sequences of $T$. culicavium were obtained for five mosquito isolates (Culex pipiens: CUL6, CUL24, CUL28 and CUL31; C. modestus: CUL30) and two bird isolates (collared flycatchers, Ficedula albicollis; PAS99 and PAS109). The trees were rooted using monoxenous trypanosomatid sequences as an outgroup. Sequences obtained in this study are underlined; accession numbers and strain and source details (where known) are given in Supplementary Table S1.

Presumable natural vertebrate host. Insectivorous songbirds (Passeriformes), e.g. collared flycatcher Ficedula albicollis (Temminck, 1815).

Experimental vertebrate host. Canary Serinus canaria (Linnaeus, 1758).
Type locality. Southern Moravia (Milovický forest game preserve, $48^{\circ} 50^{\prime} \mathrm{N} 16^{\circ} 42^{\prime} \mathrm{E}, 290 \mathrm{~m}$ above sea level).

Type isolate. CUL1 (ICUL/CZ/1998/CUL1), deposited in the collection of the Department of Parasitology, Faculty of Science, Charles University in Prague, Prague, Czech 
Republic, and in the collection of the Academy of Sciences, Biology Centre, Institute of Parasitology, České Budějovice, Czech Republic.

Syntype slides. Giemsa-stained slides of isolate CUL1, both insect and culture forms, deposited in the collection of the Department of Parasitology, Faculty of Science, Charles University in Prague, Prague, Czech Republic.

Etymology. The epithet culicavium (cu.li.ca'vi.um) is derived from a name of the insect (vector) and vertebrate hosts. L. n. culex -icis a gnat, midge and also a scientific genus name (Culex); L. gen. pl. n. avium of birds; N.L. gen. pl. n. culicavium of Culex of birds.

\section{ACKNOWLEDGEMENTS}

This research was supported by grants from the Ministry of Education of the Czech Republic (0021620828 and LC06009), the Czech Science Foundation (206/09/H026) and the Grant Agency of the Charles University (B-BIO 24109/2009). We would like to express our thanks to Jiř́ Zídek for programming the Grmeasure software. We also appreciate help from Ondra Cerný and Petr Voříšek with laboratory and fieldwork and J. Vávra for comments on the manuscript.

\section{REFERENCES}

Baker, J. R. (1956). Studies on Trypanosoma avium Danilewsky 1885. II. Transmission by Ornithomyia avicularia L. Parasitology 46, 321334.

Baker, J. R. (1966). Studies on Trypanosoma avium. IV. The development of infective metacyclic trypanosomes in cultures grown in vitro. Parasitology 56, 15-19.

Baker, J. R. (1976). Biology of the trypanosomes of birds. In Biology of the Kinetoplastida, vol. 1, pp. 131-174. Edited by W. H. Lumsden \& D. A. Evans. London: Academic Press.

Bennett, G. F. (1961). On the specificity and transmission of some avian trypanosomes. Can J Zool 39, 17-33.

Bennett, G. F. (1970). Development of trypanosomes of the T. avium complex in the invertebrate host. Can J Zool 48, 945-957.

Bennett, G. F., Whiteway, M. \& Woodworth-Lynas, C. B. (1982). A Host-Parasite Catalogue of the Avian Haematozoa (Occasional Papers in Biology, vol. 5). St. John's: Memorial University of Newfoundland.

Černý, O., Votýpka, J. \& Svobodová, M. (2011). Spatial feeding preferences of ornithophilic mosquitoes, black flies, and biting midges. Med Vet Entomol 25, 104-108.

Chatterjee, D. K. (1977). Development of Trypanosoma avium bakeri Chatterjee and Ray, 1971 in Aedes albopictus and its subsequent transmission to birds. Indian J Parasitol 1, 97-100.

David, A. \& Nair, C. P. (1955). Observations on a natural (cryptic) infection of trypanosomes in sparrows (Passer domesticus Linnaeus). I. Susceptibility of birds and mammals to the trypanosomes. Indian J Malariol 9, 95-98.

Desquesnes, M. \& Dávila, A. M. R. (2002). Applications of PCR-based tools for detection and identification of animal trypanosomes: a review and perspectives. Vet Parasitol 109, 213-231.

Desser, S. S. (1977). Ultrastructural observations on the epimastigote stages of Trypanosoma avium in Simulium rugglesi. Can J Zool 55, 1359-1367.
Desser, S. S., Mclver, S. B. \& Jez, D. (1975). Observations on the role of simuliids and culicids in the transmission of avian and anuran trypanosomes. Int J Parasitol 5, 507-509.

Dirie, M. F., Ashford, R. W., Mungomba, L. M., Molyneux, D. H. \& Green, E. E. (1990). Avian trypanosomes in Simulium and sparrowhawks (Accipiter nisus). Parasitology 101, 243-247.

Hamilton, P. B., Stevens, J. R., Gaunt, M. W., Gidley, J. \& Gibson, W. C. (2004). Trypanosomes are monophyletic: evidence from genes for glyceraldehyde phosphate dehydrogenase and small subunit ribosomal RNA. Int J Parasitol 34, 1393-1404.

Hamilton, P. B., Gibson, W. C. \& Stevens, J. R. (2007). Patterns of coevolution between trypanosomes and their hosts deduced from ribosomal RNA and protein-coding gene phylogenies. Mol Phylogenet Evol 44, 15-25.

Hertig, M. \& McConnell, E. (1963). Experimental infection of Panamanian Phlebotomus sandflies with Leishmania. Exp Parasitol 14, 92-106.

Hudec, K. \& Št'astný, K. (editors) (2005). Fauna CR: Birds 2/I, II. Prague: Academia (in Czech).

Lukeš, J. \& Votýpka, J. (2000). Trypanosoma avium: novel features of the kinetoplast structure. Exp Parasitol 96, 178-181.

Miltgen, F. \& Landau, I. (1982). [Culicoides nubeculosus, an experimental vector of a new trypanosome from psittaciforms: Trypanosoma bakeri n. sp.]. Ann Parasitol Hum Comp 57, 423-428 (in French).

Molyneux, D. H., Cooper, J. E. \& Smith, W. J. (1983). Studies on the pathology of an avian trypanosome (T. bouffardi) infection in experimentally infected canaries. Parasitology 87, 49-54.

Mungomba, L. M., Molyneux, D. H. \& Wallbanks, K. R. (1989). Hostparasite relationship of Trypanosoma corvi in Ornithomyia avicularia. Parasitol Res 75, 167-174

Novy, F. G., MacNeal, W. J. \& Torrey, H. N. (1907). The trypanosomes of mosquitoes and other insects. J Infect Dis 4, 223-276.

Podlipaev, S. A. (1990). Catalogue of World Fauna of Trypanosomatidae (Protozoa) (Proceedings of the Zoological Institute of Leningrad, vol. 144). Leningrad: Nauka.

Reeves, W. K., Adler, P. H., Ratti, O., Malmqvist, B. \& Strasevicius, D. (2007). Molecular detection of Trypanosoma (Kinetoplastida: Trypanosomatidae) in black flies (Diptera: Simuliidae). Comp Parasitol 74, 171175.

Schaudinn, F. (1904). Generation und Wirtswechsel bei Trypanosoma und Spirochaeta. Arb K Gesundheitsamte 20, 387-439 (in German).

Sehgal, R. N. M., Jones, H. I. \& Smith, T. B. (2001). Host specificity and incidence of Trypanosoma in some African rainforest birds: a molecular approach. Mol Ecol 10, 2319-2327.

Svobodová, M., Zídková, L., Čepička, I., Oborník, M., Lukeš, J. \& Votýpka, J. (2007). Sergeia podlipaevi gen. nov., sp. nov. (Trypanosomatidae, Kinetoplastida), a parasite of biting midges (Ceratopogonidae, Diptera). Int J Syst Evol Microbiol 57, 423-432.

Van Dyken, M., Bolling, B. G., Moore, C. G., Blair, C. D., Beaty, B. J., Black, W. C., IV \& Foy, B. D. (2006). Molecular evidence for trypanosomatids in Culex mosquitoes collected during a West Nile virus survey. Int J Parasitol 36, 1015-1023.

Volf, P., Hajmová, M., Sádlová, J. \& Votýpka, J. (2004). Blocked stomodeal valve of the insect vector: similar mechanism of transmission in two trypanosomatid models. Int J Parasitol 34, 1221-1227.

Votýpka, J. \& Svobodová, M. (2004). Trypanosoma avium: experimental transmission from black flies to canaries. Parasitol Res 92, $147-151$. 
Votýpka, J., Oborník, M., Volf, P., Svobodová, M. \& Lukeš, J. (2002). Trypanosoma avium of raptors (Falconiformes): phylogeny and identification of vectors. Parasitology 125, 253-263.

Votýpka, J., Lukeš, J. \& Oborník, M. (2004). Phylogenetic relationship of Trypanosoma corvi with other avian trypanosomes. Acta Protozool 43, 225-231.

Votýpka, J., Seblová, V. \& Rádrová, J. (2008). Spread of the West Nile virus vector Culex modestus and the potential malaria vector Anopheles hyrcanus in central Europe. J Vector Ecol 33, 269-277.
Wallace, F. G. \& Johnson, A. (1961). The infectivity of old cultured strains of mosquito flagellates. J Insect Pathol 3, 75-80.

Woodcock, H. M. (1906). The haemoflagellates: a review of the present knowledge relating to the trypanosomes and allied forms. Q J Microsc Sci 50, 151-231.

Zídková, L., Cepicka, I., Votýpka, J. \& Svobodová, M. (2010). Herpetomonas trimorpha sp. nov. (Trypanosomatidae, Kinetoplastida), a parasite of the biting midge Culicoides truncorum (Ceratopogonidae, Diptera). Int J Syst Evol Microbiol 60, 2236-2246. 